\title{
Detection of NRAS mutation in cell-free DNA biological fluids from patients with kaposiform lymphangiomatosis
}

\author{
Michio Ozeki ${ }^{1 *}$ (D, Yoko Aoki ${ }^{*}$, Akifumi Nozawa ${ }^{1}$, Shiho Yasue ${ }^{1}$, Saori Endo ${ }^{1}$, Yumiko Hori $^{3}$, Kentaro Matsuoka ${ }^{4}$, \\ Tetsuya Niihori ${ }^{2}$, Ryo Funayama ${ }^{5}$, Matsuyuki Shirota ${ }^{6}$, Keiko Nakayama ${ }^{5}$ and Toshiyuki Fukao ${ }^{1}$
}

\begin{abstract}
Background: Kaposiform lymphangiomatosis (KLA) has recently been distinguished as a novel subtype of generalized lymphatic anomaly (GLA) with foci of spindle endothelial cells. All cases of KLA involve multiple organs and have an unfavorable prognosis. However, the molecular pathogenesis is unknown, and there are no useful biomarkers. In the present study, we performed genetic analysis to elucidate the cause of this disease and detect biomarkers for it.

Methods: We performed whole-exome sequencing of DNA samples from leukocytes and a biopsy specimen and analyzed cell-free DNA (cfDNA) from plasma and pleural effusion of patients to identify the NRAS c.182A > G (p.Q61R) mutation using the droplet digital polymerase chain reaction (ddPCR).

Results: All KLA patients (patients 1-5) had invasive and aggressive features (hemorrhagic pleural effusions, coagulation disorder, and thrombocytopenia) and characteristic findings of KLA in their pathological examinations. In whole exome sequencing for patient 1, c.182A > G missense variant (p.Q61R) in NRAS was identified in fresh frozen samples of a mass on the left chest wall at a frequency of $5 \%$ of total alleles but not in his blood leukocytes. Furthermore, the same mutation was detected in cfDNA isolated from plasma and pleural effusion by using ddPCR. ddPCR analysis of plasma/pleural effusion samples from an additional four KLA patients showed that the same mutation was detected in isolated cfDNA in three of the four, as well as in a tissue sample from one of the three plasma/effusion-positive patients that had been obtained to confirm the mutation.
\end{abstract}

Conclusion: These results provide the first evidence that NRAS oncogenic variant was identified in DNA samples from KLA patients from not only two affected lesions but also plasma and pleural effusion.

Keywords: Vascular anomaly, Kaposiform lymphangiomatosis, Neuroblastoma RAS viral oncogene homolog, Cellfree DNA, Liquid biopsy

\section{Background}

Lymphatic anomalies (LAs), including generalized lymphatic anomaly (GLA) and kaposiform lymphangiomatosis (KLA), are extremely rare diseases with severe symptoms and poor prognosis [1]. KLA is categorized as a novel subtype of GLA in the International Society for the Study of Vascular Anomalies (ISSVA) classification

\footnotetext{
*Correspondence: michioo@gifu-u.ac.jp; aokiy@med.tohoku.ac.jp

${ }^{1}$ Department of Pediatrics, Graduate School of Medicine, Gifu University, Yanagido 1-1, Gifu 501-1194, Japan

2Department of Medical Genetics, Tohoku University School of Medicine, Sendai 980-8574, Japan

Full list of author information is available at the end of the article
}

updated in 2018 [2]. It is described as an aggressive disease of the lymphatic system and has foci of "kaposiform" abnormal spindle lymphatic endothelial cells; however, the pathogenesis of the patients remains unknown [3].

Recently, genetic research has attempted to elucidate the actual conditions and pathogenesis of vascular anomalies [4]. Low-level somatic mutations of PI-3 kinase or the RAS pathway have been detected in samples from vascular anomalies [4]. In recent studies, the NRAS c.182A > G (p.Q61R) mutation was detected in affected lesions of KLA and GLA patients $[5,6]$.

(c) The Author(s). 2019 Open Access This article is distributed under the terms of the Creative Commons Attribution 4.0 International License (http://creativecommons.org/licenses/by/4.0/), which permits unrestricted use, distribution, and 
However, a tissue biopsy can lead to severe complications, such as bleeding or lymphatic leakage. Therefore, development of noninvasive diagnostic methods may not only provide a more accurate diagnosis, but also decrease the risk of complications. Analysis of cell-free DNA (cfDNA), so-called liquid biopsy, is becoming a promising clinical application for molecular testing and cancer detection [7].

Here, we describe a case of KLA, in which a somatic mutation in the NRAS gene was detected both in a biopsy specimen and in cfDNA. Additionally, the same mutation was detected in cfDNA from the four other patients with KLA and from a tissue sample obtained from one of them. Our data therefore suggest that cfDNA analysis might be a useful method for diagnosing KLA.

\section{Methods}

\section{Patients}

Medical records from January 2016 to December 2018 on 8 patients (5 with KLA and 3 with GLA) were retrospectively analyzed. This study was approved by the Ethics Committees of Gifu University School of Medicine and Tohoku University School of Medicine (25-136 and 2013-1-278). The patients or their legal guardians gave their informed consent to be included. Data on patient characteristics, diagnosis, clinical symptoms, and test results were collected.

\section{Genetic analysis of a patient with KLA (patient 1)}

Genomic DNA was extracted from blood samples using standard protocols. DNA was extracted from frozen samples of a mass on the left chest wall of patient 1 using Qiagen DNeasy Blood and Tissue Kit.

In whole-exome sequencing (WES), targeted enrichment was performed using the SureSelect Human All Exon V6 kit. Exon-enriched DNA libraries were sequenced on the Illumina Hiseq 2500 for $101 \mathrm{bp}$. Burrows-Wheeler Alignment (BWA) was used to align the sequence reads to the human genome (hg19); all parameters of BWA were kept at the default settings. Following removal of duplicates from the alignments, realignment around known indels, recalibration, and SNP/ indel calling were performed with the UnifiedGenotyper tool of the Genome Analysis Toolkit (GATK) (1.5) [8]. ANNOVAR was used for the annotation against the RefSeq database and dbSNP [9]. Novel mutations were extracted according to the variants located in an exon or splice site, excluding synonymous variants, and variants that exhibited an allele frequency of less than $1 \%$ or were not reported in variant databases in the 1000 Genomes Project (http://browser.1000genomes.org/), the Exome Aggregation Consortium (ExAC, http://exac.broadinstitute.org/), the genome aggregation database (gnomAD, https:/gnomad.broadinstitute.org/), the Human Genome
Variation Database (HGVD, http://www.genome.med. kyoto-u.ac.jp/SnpDB/), and the Integrative Japanese Genome Variation Database (iJGVD, https://ijgvd.mega bank.tohoku.ac.jp/). Detected variants were confirmed through visual examination of the genetic data using the Integrative Genomics Viewer (IGV; http://www.broadin stitute.org/igv/).

To identify low-frequency somatic mutations in frozen samples of a mass on the left chest wall, variants were called by MuTect. Identified variations were visually reviewed by IGV.

PCR products of exon 2 in NRAS were subcloned using a pTOPO TA cloning kit (Invitrogen, Carlsbad, CA) and transformed in TOP10F competent cells (Invitrogen). Plasmids were purified from each colony and sequenced.

\section{Droplet digital polymerase chain reaction (ddPCR) analysis on NRAS p.Q61R mutation}

CfDNA was extracted using a QIAamp Circulating Nucleic Acid Kit (Qiagen). Extracted DNA was quantified using a Qubit ${ }^{\oplus}$ 3.0 Fluorometer (Thermo Fisher Scientific). Genomic DNA was extracted from formalin-fixed, paraffin-embedded (FFPE) tissue using a Maxwell RSC DNA FFPE Kit (Promega). NRAS mutation detection assays were performed using the mutation detection assay NRAS p.Q61R c.182A > G (Bio-Rad) in a ddPCR apparatus (QX200 ${ }^{\mathrm{mm}}$ AutoDG $^{\mathrm{ma}}$ Droplet Digital $^{\mathrm{mm}}$ PCR system; Bio-Rad). Each ddPCR assay was performed in triplicate for each sample (patients 1-8). We used BioRad QuantaSoft Analysis Pro for data analysis. The data are expressed as a percentage of mutant droplets relative to the sum of wild-type droplets of each sample. The experiment featured a positive control, which included only wild-type DNA, and a negative control, which did not include DNA. The genome of the human skin melanoma cell line (SK-MEL-2) was used as a positive control as it is known to have an NRAS Q61R mutation.

\section{Results \\ Case description \\ Patient 1 (P1)}

A 19-year-old male suffered from hemoptysis, dyspnea, and gastrointestinal hemorrhage. He had had a subcutaneous mass at the left chest wall from birth. The patient had previously been admitted to another hospital for examination and management. The examination revealed pleural effusion, multiple osteolytic lesions of the spine, and a splenic cystic lesion. The patient had been diagnosed with lymphangiomatosis and treated with conservative therapy; however, his symptoms did not improve. Two years later, he was therefore transferred to Gifu University Hospital for alternative treatment. Physical examination revealed a reddish brown, soft, and 
nontender subcutaneous mass $(10 \times 8 \mathrm{~cm})$ on the left chest wall with no signs of inflammation (Fig. 1a). Plain radiography and gadolinium-enhanced magnetic resonance imaging (MRI) of the chest revealed pleural effusion, an infiltrative soft-tissue mass at the left chest wall, and a retroperitoneal lesion (Fig. 1b-d). Laboratory data indicated mild thrombocytopenia $\left(9.0 \times 10^{4} / \mu \mathrm{L}\right)$ and coagulopathy (D-dimer: $31.0 \mu \mathrm{g} / \mathrm{ml}$, fibrinogen: $181 \mathrm{mg} /$ $\mathrm{dL}$ (reference range for D-dimer is $<0.5 \mu \mathrm{g} / \mathrm{ml}$ and for fibrinogen $200-400 \mathrm{mg} / \mathrm{dL}$ ). Colonoscopy showed bleeding and multiple dilated tortuous blood vessels running along the surface of the sigmoid colon. An open biopsy specimen of the subcutaneous lesion showed irregularly dilated endothelial cells and foci of spindle cells (Fig. 1e). In immunohistochemical study, both endothelial cells and spindle cells showed positive reactivity for CD31, D2-40, and Prox-1, identifying them as being of lymphatic origin (Fig. 1f). Following these results, the diagnosis of KLA was made. We decided to undertake treatment using a mammalian target of rapamycin (mTOR) inhibitor, sirolimus. The treatment was approved by the review board of Gifu University Hospital and written informed consent was obtained from the patient. Sirolimus treatment was started at $2 \mathrm{mg} /$ day. Dose adjustments were made to maintain the drug at a therapeutic level of 5 to $15 \mathrm{ng} / \mathrm{ml}$. Within 2 weeks of starting the sirolimus treatment, pleural effusion and gastrointestinal hemorrhage decreased and the lesions of the chest wall shrank. After 6 months, the coagulopathy improved (D-dimer: $11.2 \mu \mathrm{g} / \mathrm{ml}$, fibrinogen: $260 \mathrm{mg} / \mathrm{dL}$ ); however, the mild thrombocytopenia did not change. At the time of writing, the patient has been continuing therapy for 2 years without any adverse effects.

\section{Patients 2-8 (P2-8)}

Patients 2, 3, 4, and 5 (P2-5) were diagnosed with KLA based on clinical and pathological findings (Table 1). All patients had invasive and aggressive features and characteristic findings of KLA (foci of spindle cells) in their pathological examinations. In contrast, patients 6,7 , and 8 (P6-8) were diagnosed with GLA because they did not have characteristic evidence of KLA. Only one patient in the P2-8 group (P2) had undergone genetic analysis of the affected lesions because we did not have fresh frozen samples from the others (P3-8).

\section{WES and cfDNA analysis in patient 1}

WES analysis was performed on DNA from leukocytes and biopsy specimens from the chest wall mass of patient 1 (P1). We first compared the variants between the

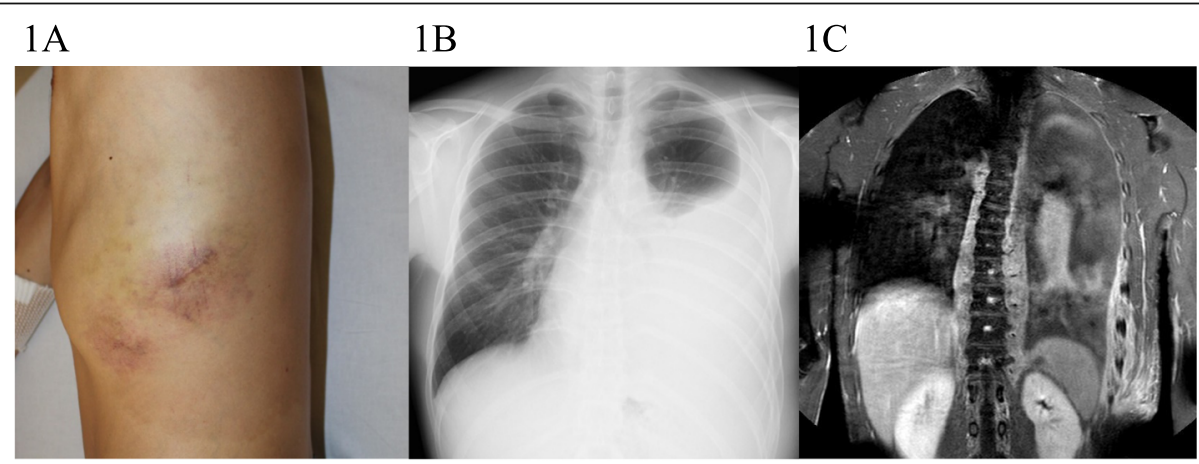

1D

$1 \mathrm{E}$

$1 \mathrm{~F}$

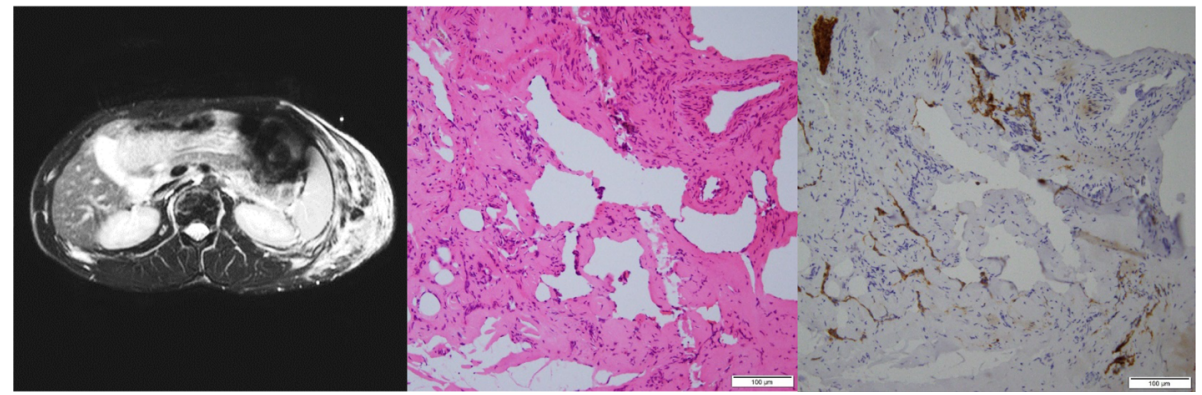

Fig. 1 Clinical appearance and examinations of the patient with KLA (P1). a Cutaneous manifestation of left chest wall. b Chest radiography shows pleural effusion and mediastinal enlargement. c, d Chest contrast-enhanced MRI demonstrates diffuse thickening of the left chest wall, pleural effusion in the left lung, and retroperitoneal soft-tissue mass. e, f Specimen shows proliferation of thin-walled, anastomosing lymphatic vessels lined by a single layer of endothelial cells with a focus of spindle cells (bar $100 \mu \mathrm{m}$, H\&E). Endothelial cells were identified as lymphatics using D2-40 (bar $100 \mu \mathrm{m}$ ) 


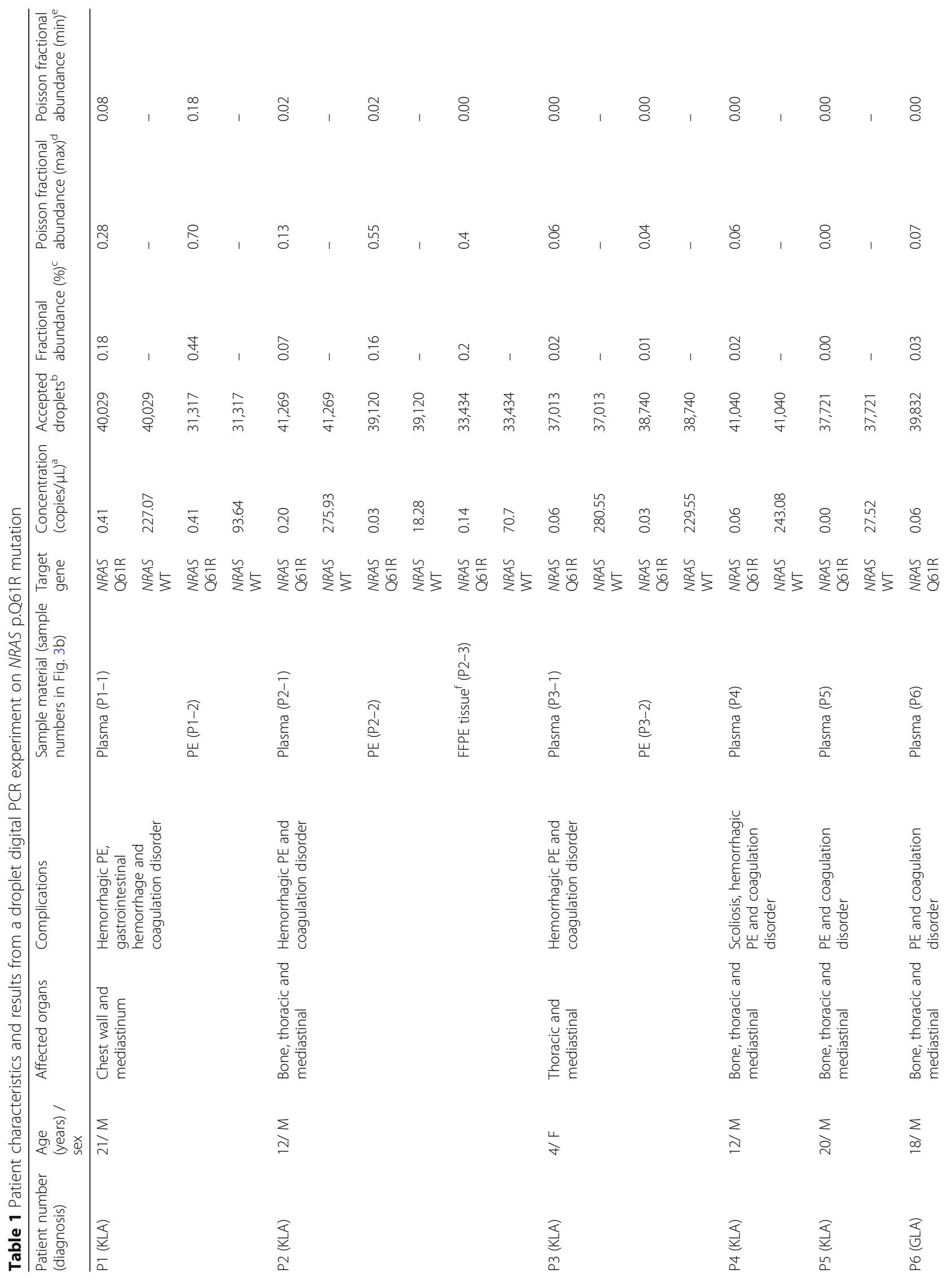




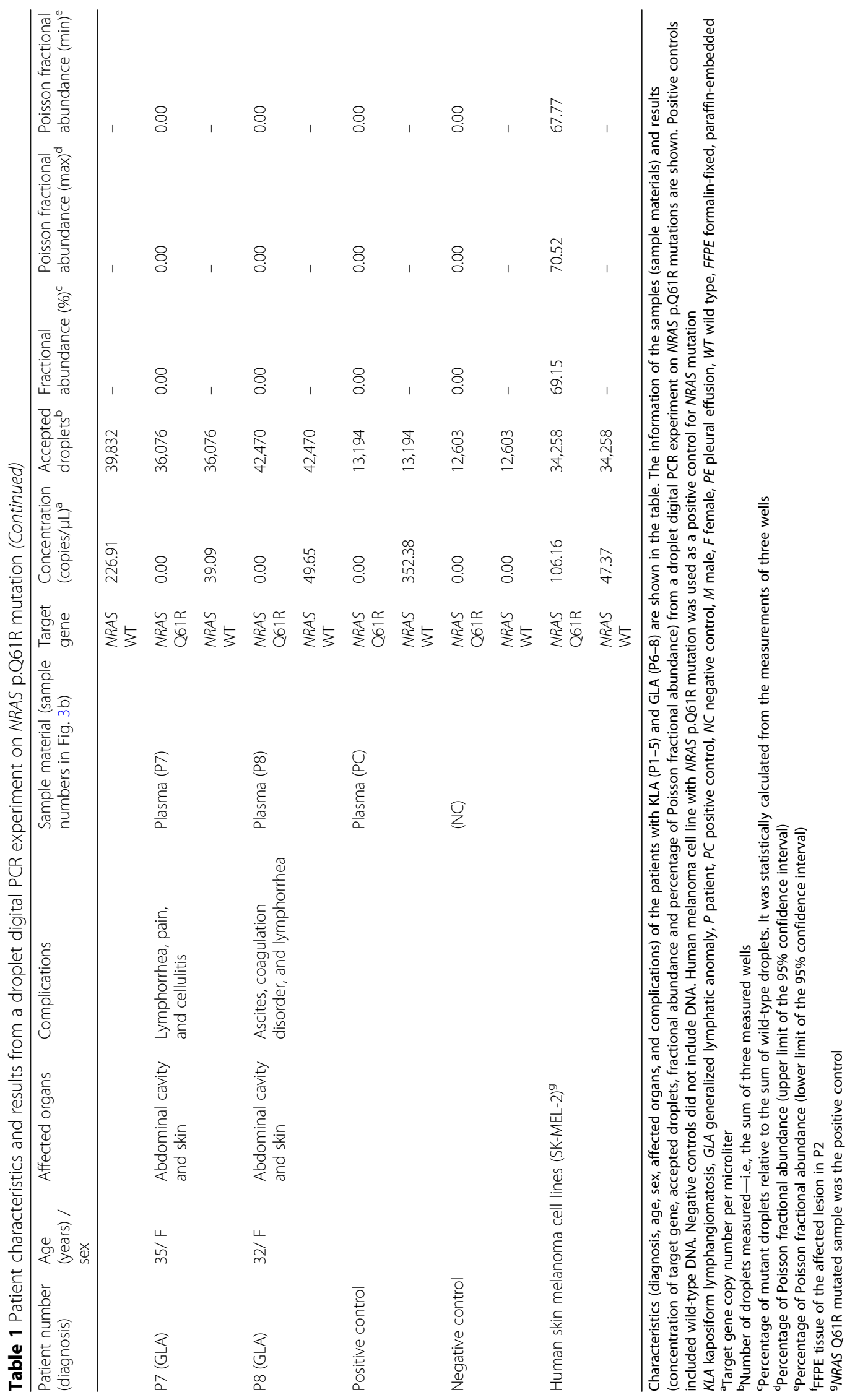


tumor samples and the blood samples using data from the BWA-GATK pipeline. No germline or somatic variants that could be associated with lymphatic abnormalities were identified. We then filtered less frequent variants that were specifically identified in the chest mass samples using MuTect software. Of those, the NRAS c.182A > G variant (p.Q61R) was identified in 9/ 176 alleles (5\%) of the tumor samples, while no mutant reads were detected in the leukocyte samples (Fig. 2a). We then performed PCR using primers that amplify exon 2 of NRAS. Subcloning and Sanger sequencing of the PCR product identified the c. $182 \mathrm{~A}>\mathrm{G}$ variant in 6 of 60 clones (10\%) (Fig. 2b). These results suggested the presence of a low-frequency somatic NRAS variant in tumor tissues. Furthermore, the cfDNA was extracted from the plasma $(5 \mathrm{ml})$ and pleural effusion $(5 \mathrm{ml})$ (790.4 ng and $93.8 \mathrm{ng}$, respectively). NRAS c.182A > G mutation was detected at a low frequency in cfDNA isolated from plasma and pleural effusion using ddPCR (Fig. 3a, b and Table 1).

\section{cfDNA analysis in plasma and pleural effusions from other KLA and GLA patients}

We performed cfDNA analysis in the plasma and pleural effusion of KLA (P2-5) and GLA patients (P6-8). We obtained pleural effusion samples from P2 and P3 (P2-2 and P3-2). In cfDNA analysis, a low-frequency NRAS p.Q61R mutation was detected in both plasma and pleural effusion samples of P2 and P3 (P2-1, P2-2, P31 , and P3-2) and plasma samples of P4 and P6. Additionally, a low-frequency NRAS p.Q61R mutation was detected in FFPE tissue of the affected bone lesion in P2 (P2-3) (Fig. 3b and Table 1). P3-8 had not undergone genetic analysis of the lesions to determine whether the same NRAS mutation could be detected from these samples.

\section{Discussion}

We here present the first study of liquid biopsy for NRAS mutation in KLA cases. In a first case with intractable KLA treated with sirolimus, which induced improvement of symptoms and tumor regression, WES of DNA samples from leukocytes and tumor tissues showed the presence of NRAS c.182A > G (p.Q61R) in $5 \%$ of alleles in tumor tissues, which was confirmed by TA cloning of the PCR product followed by sequencing. The NRAS mutation was also detected in cfDNA isolated from plasma and pleural effusion. Furthermore, we conducted target-specific NRAS mutation analysis by using cfDNA isolated from liquid sample of 7 patients (4KLA and 3GLA) and detected the targeted gene mutation in $80 \%(4 / 5)$ of KLA patients (P1, P2, P3, and P4). The genotyping of FFPE tissue showed that the same mutation was also detected in an affected lesion from a KLA patient (P2). These results suggested that this somatic oncogenic NRAS mutation may be involved in the pathogenesis of KLA, and cfDNA might be useful for diagnosing KLA.

KLA is recognized as a new entity that has an aggressive course and poor prognosis. Recent research has attempted to elucidate the actual conditions and pathogenesis of these diseases. KLA was classified by the World Health Organization as a rare tumor of lymphatic vessel origin with the capacity to metastasize [10]. Although the ISSVA classification of 2014 categorized this condition as a provisionally unclassified vascular anomaly, KLA was categorized as a subtype of GLA in the ISSVA classification updated in 2018 [2]. The key

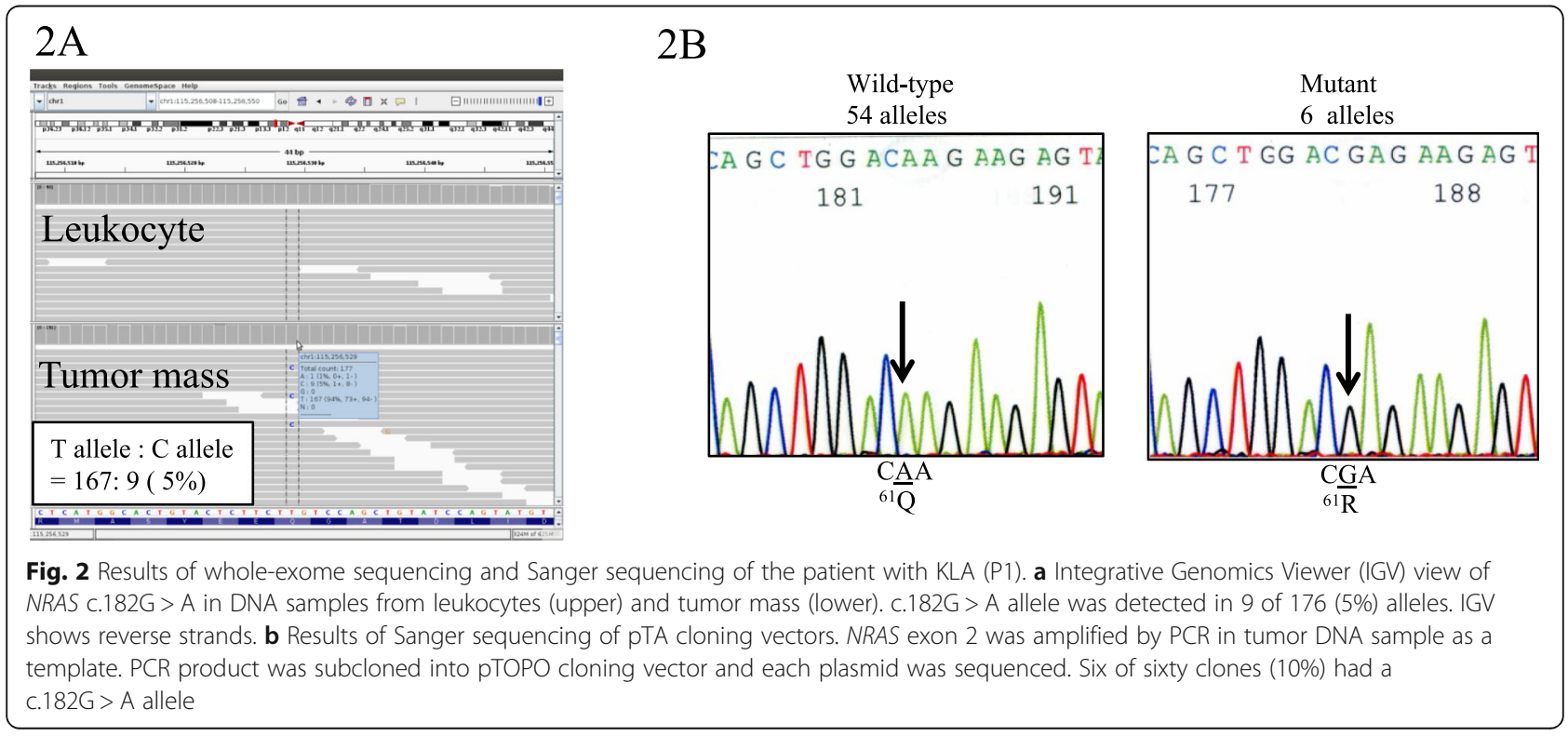



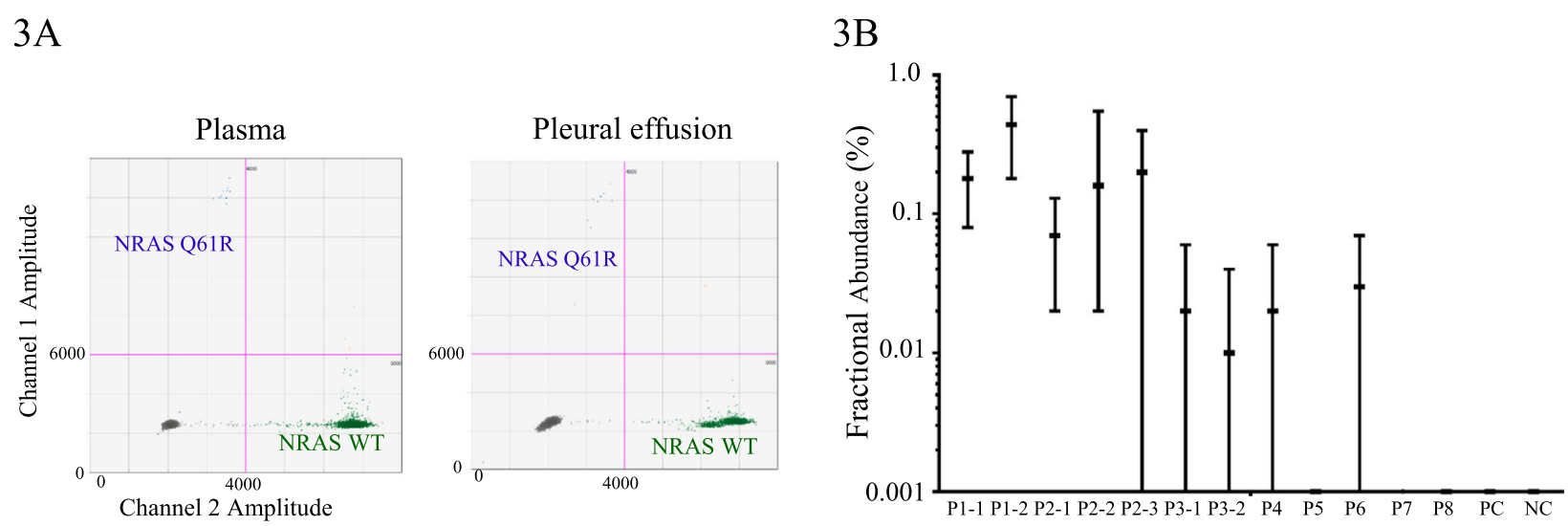

The name of sample

Fig. 3 Cell-free DNA analysis of GLA and KLA patients. a Gene multiplexing on a droplet digital polymerase chain reaction (ddPCR) system using probes that target specific NRAS mutation in cell-free DNA isolated from plasma and pleural effusion samples (P1). NRAS mutation detection assays were performed using the mutation detection assay NRAS p.Q61R c.182A > G (Bio-Rad) in a ddPCR apparatus (QX200 ${ }^{\text {TM }}$ AutoDG ${ }^{\text {TM }}$ Droplet Digital $^{\text {TM }}$ PCR system; Bio-Rad). Two fluorescence amplitude bands were clearly generated for each gene (upper band: NRAS Q61R mutant, lower band: NRAS wild type). $\mathbf{b}$ The data are expressed as a percentage of mutant droplets relative to the sum of wild-type droplets of each sample (P1-8, positive control and negative control). Positive controls included wild-type DNA. Negative control did not include DNA. Data are shown as the median and 95\% confidence interval. The identities of the samples are as follows: P1-1: plasma sample of P1, P1-2: PE sample of P1, P2-1: plasma sample of P2, P2-2: PE sample of P2, P2-3: FFPE sample of P2, P3-1: plasma sample of P3, P3-2: PE sample of P3, P4-8: plasma sample of P4-8, PC: plasma sample of PC, NC: plasma sample of NC. WT: wild type, PE: pleural effusion, PC: positive control, NC: negative control

signals of KLA are thrombocytopenia, coagulation disorder, and hemorrhagic pericardial and pleural effusion or ascites. However, the pathogenesis and etiology of KLA are still unknown. GLA also involves the diffuse or multicentric proliferation of lymphatic vessels in several organ systems, and an appropriate diagnosis is difficult because the clinical findings of KLA and GLA overlap [1]. Dilated malformed lymphatic channels lined by a single layer of endothelial cells are common to both GLA and KLA; the latter also has foci of patternless clusters of intra- or peri-lymphatic spindle cells associated with platelet microthrombi, extravasated red blood cells, hemosiderin, and some degree of fibrosis. Recently, two reports on cytokine analysis in patients with lymphatic anomalies showed that angiopoietin-2 and some cytokines are important markers for KLA [11, 12]. However, differentiation of these diseases is challenging based on their phenotypic presentation alone, so further study is needed.

Although genetic analysis revealed somatic mutations in genes associated with the phosphoinositide 3-kinase (PI3K) pathway in patients with LAs [4], little has been reported on the associated genetic abnormalities of KLA. In a recent study, Manevitz-Mendelson et al. reported the possibility that somatic NRAS mutation causes GLA [6]. Activating mutations in RAS proto-oncogenes (KRAS, HRAS, and NRAS) have been found in a variety of human malignancies, suggesting a dominant role in carcinogenesis [13-15]. The authors isolated lymphangiomatosis endothelial cells from a GLA patient using
CD31-coated magnetic beads and identified a somatic activating mutation in NRAS in fewer than $30 \%$ of the alleles of the endothelial cells. The results of the study showed that the NRAS mutation plays key roles in the regulation of angiogenesis and lymphangiogenesis. Barclay et al. reported that NRAS mutations were detected in the affected lesions of KLA patients [5]. We also found that KLA patients possessed the same NRAS mutation. Although why the same NRAS mutation could be associated with both GLA and KLA is not clear, it could be speculated that the confusion is due to not only the biology of these diseases but also the difficulty of the diagnosis. The pathogenesis of these diseases requires further investigation.

In a wide spectrum of RAS-related disorders, recently called RASopathies [16], somatic $R A S$ mutations, especially somatic NRAS mutation at codon 61 (p.Q61R/K), have been identified in a variety of human malignancies [15]. Somatic NRAS c.182A $>$ G (p.Q61R) mutations have also been identified in nonmalignant cancers, including pyogenic granuloma [17] and Langerhans cell histiocytosis [18]. In contrast, germline NRAS mutations have been identified in patients with Noonan syndrome, which is characterized by short stature, congenital heart disease, lymphatic abnormalities, chest deformity, and predisposition to malignant tumors [16]. However, NRAS p.Q61R mutation has never been identified in Noonan syndrome patients, suggesting that patients with germline p.Q61R mutation do not survive to birth because of its strong activation of the downstream 
pathway. In mosaic RASopathies, NRAS p.Q61R mutations have been identified in a patient with Schimmelpenning syndrome [19], patients with neurocutaneous melanosis [20], and patients with cutaneous-skeletal hypophosphatemia syndrome [21]. These results suggest that somatic or mosaic NRAS p.Q61R mutations cause a broad spectrum of disorders, which depend on the lineages or cells in which they occur.

Low-frequency NRAS mutation was identified in a chest mass in our patient. However, we were not able to identify in which cells this mutation arose. NRAS mutations play a critical role in angiogenesis and lymphangiogenesis [6]. We also performed ddPCR assays of the NRAS p.Q61R mutation for other KLA and GLA patients who had not undergone WES (genetic) analysis because we did not have fresh frozen samples of their affected lesions. A total of $80 \%(4 / 5)$ of patients with KLA and $33.3 \%(1 / 3)$ of patients with GLA showed lowfrequency NRAS mutation in cfDNA isolated from plasma or pleural effusion. Additionally, the same mutation was detected in the FFPE tissue of another KLA patient (P2). Although the results of P3, 4, and 6 could be false positives because the minimum Poisson fractional abundance in their samples was statistically 0.0, the NRAS p.Q61R mutation was apparently detected in tissue samples from two KLA patients (P1 and P2). Our results indicate the possibility of developing a novel diagnostic method, a so-called liquid biopsy, for KLA without the need for an invasive procedure.

The finding that RAS mutation drives vascular anomalies including GLA and KLA provides potential opportunities to develop targeted therapies for current drug-resistant lesions. Treatment with an mTOR inhibitor, sirolimus, and an MEK inhibitor, trametinib, had an effect of reducing the viability of the affected cells through inhibition of the phosphorylation of AKT and extracellular signal-regulated kinase (ERK) [6]. NRAS inhibition by these drugs might be a promising option for the treatment of these lymphatic anomalies. In our patient, sirolimus was effective to improve clinical symptoms and subcutaneous lesions. These genes are associated with the pathogenic etiology of lymphatic diseases and their inhibition might thus be a target for treatment.

\section{Conclusions}

Our results provide the first evidence of an NRAS oncogenic variant in cfDNA in two patients with KLA. Furthermore, this is the first time that mutated gene from isolated cfDNA has been detected in patients with vascular anomalies. The observation that KLA can be caused by mutations in NRAS offers insight into the basic biology of KLA. It suggests that KLA is a group of RASopathies, which may facilitate efforts to develop an appropriate therapy for it. Furthermore, liquid biopsy using cfDNA could be a new useful method for these diseases.

\section{Abbreviations}

BWA: Burrows-Wheeler Alignment; cfDNA: Cell-free DNA; ddPCR: Droplet digital polymerase chain reaction; ERK: Extracellular signal-regulated kinase; ExAC: Exome Aggregation Consortium; FFPE: Formalin-fixed, paraffinembedded; GATK: Genome Analysis Toolkit; GLA: Generalized lymphatic anomaly; HGVD: Human Genome Variation Database; IGV: Integrative Genomics Viewer; iJGVD: Integrative Japanese Genome Variation Database; ISSVA: International Society for the Study of Vascular Anomalies; KLA: Kaposiform lymphangiomatosis; LA: Lymphatic anomaly; MRI: Magnetic resonance imaging; mTOR: Mammalian target of rapamycin; P: Patient; PI3K: Phosphoinositide 3-kinase; WES: Whole-exome sequencing

\section{Acknowledgments}

We thank Dr. Keigo Osuga and Dr. Taro Koba of Osaka University, and Dr. Kaori Kanda, Dr. Akihito Nagano, and Dr. Yusaku Miyamoto of Gifu University for their helpful comments. We thank Miyuki Tsuda, Mami Kikuchi, Makiko Nakagawa, Yoko Tateda, and Kiyotaka Kuroda in Tohoku University for providing technical assistance of WES analysis. We also acknowledge the technical support of the Biomedical Research Core of Tohoku University Graduate School of Medicine. We also thank Edanz (www.edanzediting.co.jp) for editing the English text of a draft of this manuscript. The ddPCR assay of cfDNA was performed by RIKEN GENESIS Co., Ltd. (Kanagawa, Japan).

\section{Authors' contributions}

$M O, F T$, and YA conceived the study and participated in its design. MO, AN, $\mathrm{SY}$, and SE provided medical care for the patients and collected data. $\mathrm{YH}$ and KM performed the pathological analysis. TN, RF, MS, KN, and YA performed the WES and genetic analysis of the patients. MO, FT, and YA wrote the manuscript. All authors read and approved the final manuscript.

\section{Funding}

The present study was supported in part by the Clinical Research-Clinical Trial Promotion Research Project (18lk0201055h0003) and Practical Research Project for Rare/Intractable Diseases (18ek0109277h0002 and 18ek0109278h0002) from Japan's Agency for Medical Research and Development, AMED.

\section{Availability of data and materials}

The datasets and analysis performed during the current study are available from the corresponding author upon reasonable request.

\section{Ethics approval and consent to participate}

This study was approved by Ethics Committees of the Gifu University School of Medicine and Tohoku University School of Medicine. The patients or their legal guardians gave their informed consent to be included.

\section{Consent for publication}

The patients or their legal guardians provided written informed consent for the publication of this study.

\section{Competing interests}

$\mathrm{MO}$ and TF received research funding from Nobelpharma. Sirolimus tablets were supplied by Nobelpharma. The other authors declare no competing interests.

\section{Author details}

1'Department of Pediatrics, Graduate School of Medicine, Gifu University, Yanagido 1-1, Gifu 501-1194, Japan. ${ }^{2}$ Department of Medical Genetics, Tohoku University School of Medicine, Sendai 980-8574, Japan. ${ }^{3}$ Department of Pathology, Graduate School of Medicine, Osaka University, Osaka 565-0871, Japan. ${ }^{4}$ Department of Pathology, Dokkyo Medical University Saitama Medical Center, Saitama 343-8555, Japan. ${ }^{5}$ Division of Cell Proliferation, United Centers for Advanced Research and Translational Medicine, Tohoku University Graduate School of Medicine, Sendai 980-8575, Japan. ${ }^{6}$ Division of Interdisciplinary Medical Science, United Centers for Advanced Research and Translational Medicine, Tohoku University Graduate School of Medicine, Sendai 980-8575, Japan. 
Received: 14 January 2019 Accepted: 4 September 2019

Published online: 11 September 2019

\section{References}

1. Ozeki M, Fujino A, Matsuoka K, Nosaka S, Kuroda T, Fukao T. Clinical features and prognosis of generalized lymphatic anomaly, Kaposiform Lymphangiomatosis, and Gorham-stout disease. Pediatr Blood Cancer. 2016; 63:832-8.

2. International Society for the Study of Vascular Anomalies: ISSVA classification for Vascular Anomalies (approved at the May 2018 General Assembly in Amsterdam, the Netherlands). https://www.issva.org/ classification. Accessed 2 Jan 2019.

3. Croteau SE, Kozakewich HP, Perez-Atayde AR, Fishman SJ, Alomari Al, Chaudry G, Mulliken JB, Trenor CC 3rd. Kaposiform lymphangiomatosis: a distinct aggressive lymphatic anomaly. J Pediatr. 2014;164:383-8.

4. Greene AK, Goss JA. Vascular anomalies: from a Clinicohistologic to a genetic framework. Plast Reconstr Surg. 2018;141:709e-17e.

5. Barclay SF, Inman KW, Luks VL, McIntyre JB, Al-Ibraheemi A, Church AJ, Perez-Atayde AR, Mangray S, Jeng M, Kreimer SR, Walker L, Fishman SJ, Alomari Al, Chaudry G, Trenor CC III, Adams D, Kozakewich HPW, Kurek KC. A somatic activating NRAS variant associated with kaposiform lymphangiomatosis. Genet Med. 2019;21:1517-24.

6. Manevitz-Mendelson E, Leichner GS, Barel O, Davidi-Avrahami I, ZivStrasser L, Eyal E, Pessach I, Rimon U, Barzilai A, Hirshberg A, Chechekes K, Amariglio N, Rechavi G, Yaniv K, Greenberger S. Somatic NRAS mutation in patient with generalized lymphatic anomaly. Angiogenesis. 2018;21:287-98.

7. Corcoran RB, Chabner BA. Application of cell-free DNA analysis to Cancer treatment. N Engl J Med. 2018;379:1754-65.

8. McKenna A, Hanna M, Banks E, Sivachenko A, Cibulskis K, Kernytsky A, Garimella K, Altshuler D, Gabriel S, Daly M. The genome analysis toolkit: a MapReduce framework for analyzing next-generation DNA sequencing data. Genome Res. 2010;20:1297-303.

9. Wang K, Li M, Hakonarson H. ANNOVAR: functional annotation of genetic variants from high-throughput sequencing data. Nucleic Acids Res. 2010;38: e164.

10. Fletcher CDM, Bridge JA, Hogendoorn P, Mertens F. WHO classification of tumours of soft tissue and bone, vol. 5. 4th ed. Geneva: WHO; 2013. p. 137.

11. Le Cras TD, Mobberley-Schuman PS, Broering M, Fei L, Trenor CC 3rd, Adams DM. Angiopoietins as serum biomarkers for lymphatic anomalies. Angiogenesis. 2017;20:163-73.

12. Ozeki M, Nozawa A, Kawamoto N, Fujino A, Hirakawa S, Fukao T. Potential biomarkers of kaposiform lymphangiomatosis. Pediatr Blood Cancer. 2019; 66:e27878.

13. Bourdeaut F, Herault A, Gentien D, Pierron G, Ballet S, Reynaud S, Paris R, Schleiermacher G, Baumann C, Philippe-Chomette P, GauthierVillars M, Peuchmaur M, Radvanyi F, Delattre O. Mosaicism for oncogenic G12D KRAS mutation associated with epidermal nevus, polycystic kidneys and rhabdomyosarcoma. J Med Genet. 2010;47: 859-62.

14. Aoki $Y$, Niihori T, Narumi Y, Kure S, Matsubara Y. The RAS/MAPK syndromes: novel roles of the RAS pathway in human genetic disorders. Hum Mutat. 2008;29:992-1006.

15. Khan AQ, Kuttikrishnan S, Siveen KS, Prabhu KS, Shanmugakonar M, AlNaemi HA, Haris M, Dermime S, Uddin S. RAS-mediated oncogenic signaling pathways in human malignancies. Semin Cancer Biol. 2019;54:1-13. https:// doi.org/10.1016/j.semcancer.2018.03.001. Epub 2018 Mar 7.

16. Aoki Y, Niihori T, Inoue S, Matsubara Y. Recent advances in RASopathies. J Hum Genet. 2016:61:33-9.

17. Groesser L, Peterhof E, Evert M, Landthaler M, Berneburg M, Hafner C. BRAF and RAS mutations in sporadic and secondary pyogenic granuloma. J Invest Dermatol. 2016;136:481-6.

18. Mourah S, How-Kit A, Meignin V, Gossot D, Lorillon G, Bugnet E, Mauger F, Lebbe C, Chevret S, Tost J, Tazi A. Recurrent NRAS mutations in pulmonary Langerhans cell histiocytosis. Eur Respir J. 2016;47:1785-96.

19. Kuroda Y, Ohashi I, Enomoto Y, Naruto T, Baba N, Tanaka Y, Aida N, Okamoto N, Niihori T, Aoki Y, Kurosawa K. A postzygotic NRAS mutation in a patient with Schimmelpenning syndrome. Am J Med Genet A. 2015;167: 2223-5

20. Kinsler VA, Thomas AC, Ishida M, Bulstrode NW, Loughlin S, Hing S, Chalker J, McKenzie K, Abu-Amero S, Slater O, Chanudet E, Palmer R,
Morrogh D, Stanier P, Healy E, Sebire NJ, Moore GE. Multiple congenital melanocytic nevi and neurocutaneous melanosis are caused by postzygotic mutations in codon 61 of NRAS. J Investig Dermatol. 2013; 133:2229-36.

21. Lim YH, Ovejero D, Derrick KM, Collins MT, Choate KA. Cutaneous skeletal hypophosphatemia syndrome (CSHS) is a multilineage somatic mosaic RASopathy. J Am Acad Dermatol. 2016;75:420-7.

\section{Publisher's Note}

Springer Nature remains neutral with regard to jurisdictional claims in published maps and institutional affiliations.
Ready to submit your research? Choose BMC and benefit from:

- fast, convenient online submission

- thorough peer review by experienced researchers in your field

- rapid publication on acceptance

- support for research data, including large and complex data types

- gold Open Access which fosters wider collaboration and increased citations

- maximum visibility for your research: over $100 \mathrm{M}$ website views per year

At $\mathrm{BMC}$, research is always in progress.

Learn more biomedcentral.com/submissions 